\title{
TEMPERATURE EFFECT ON FIBER OPTICAL SENSORS FOR DRY CAST STORAGE HEALTH MONITORING
}

\author{
Bin Lin, Lingyu Yu, Victor Giurgiutiu \\ University of South Carolina \\ Columbia, SC 29208, USA \\ linbin@cec.sc.edu
}

\begin{abstract}
The increasing number, size, and complexity of nuclear facilities deployed worldwide are increasing the need to maintain readiness and develop innovative sensing materials to monitor important to safety structures (ITS). Assessing and supporting next generation nuclear materials management and safeguards for future U.S. fuel cycles with minimum human intervention is of paramount importance. Technologies for the diagnosis and prognosis of a nuclear system, such as dry cast storage system (DCSS), can improve verification of the health of the structure that can eventually reduce the likelihood of inadvertently failure of a component. In the past decades, an extensive sensor technology development has been used for structural health monitoring (SHM). Fiber optical sensors have emerged as one of the major SHM technologies developed particularly for temperature and strain measurements. However, the fiber optical sensors and sensing system has not been developed with adequate solutions and guideline for DCSS applications. This paper presents an experimental study of temperature effect on fiber Bragg grating (FBG) sensors. The reflective spectrum of FBG sensors on the structure was measured with a tunable laser source. The shift of FBG reflective spectrum reflected the thermal expansion on the structure. The shift of the spectrum due to the temperature effect was correlated to the temperature changes. In addition, the FBG sensing methodology including high frequency guided ultrasonic waves (GUW) under different temperatures were also performed to check the performance of high frequency, small strain sensing. The potential of FBG sensors for DCSS applications was explored. The paper ends with conclusions and suggestions for further work.
\end{abstract}

\section{INTRODUCTION}

Many pressure vessel and piping (PVP) systems are safetycritical facilities in need of monitoring over prolonged periods of time. Structural health monitoring (SHM) is an emerging technology that aims at monitoring the state of a structure through the use of networks of permanently mounted sensors. SHM technologies have been developed primarily within the aerospace and civil engineering communities. However, SHM concepts and methodologies could be extended to other safetycritical systems. Many PVP applications, as for example, nuclear power plants (NPP) and nuclear dry cask storage systems (DCSS) are safety-critical facilities in need of monitoring over prolonged periods of time. One of the key aspects of improving the reliability, sustaining the safety, and extending the life of current PVP is to develop technologies that can better diagnose their state of structural health.

Monitoring these aging structures with minimum human intervention would be of paramount importance and great benefit. In addition, such tools can provide invaluable and timely information for verification of the predicted mechanical performance of critical PVP applications during off-normal occurrence and accident events such as the tsunami and earthquake event that affected Fukushima Daiichi nuclear power plant. One possible way to assess the current condition of structures, and, more importantly, to predict the residual safe operating life of PVP systems is to develop new sensing techniques that monitor the integrity of PVP components.

Fiber optical SHM technologies have a wide range of applications based on both strain and temperature sensing capability. Hence, opportunities exist for transitioning these fiber optical SHM technologies into PVP monitoring applications. By 
implementing "embedded" fiber optical sensors, SHM can enhance the inspectability of PVP systems. This will allow realtime assessment of the structural conditions and prediction of service life with greater reliability.

However, certain special operational conditions exist that are specific to PVP applications such as: aggressive environments, marine environments, radiation fields, and chemical, mechanical and thermal stressors. This will require research to develop monitoring, diagnosis, and prognosis tools that can aid to establish a strong technical basis for the safety of PVP systems. This paper addresses the issue of transitioning the fiber optical SHM concept to the monitoring of PVP applications and evaluations the opportunities and challenges associated with this process. Our focus is mainly of PVP application in the DCSS system.

\section{DRY CASK STORAGE SYSTEM SAFETY ASSESSMENT NEED}

Following the issuance of the Blue Ribbon Commission (BRC) on America's Nuclear Future Final Report in 2012 [1], interim storage of spent nuclear fuel from reactor sites has gained additional importance and urgency for resolving wastemanagement-related technical issues. In total, there are over 1482 dry cask storage system (DCSS) in use at US plants, storing 57,807 fuel assemblies. On July 12, 2011, the Nuclear Regulatory Commission (NRC) issued recommendations on how to enhance "spent fuel makeup capability and instrumentation for the spent fuel pool". This includes the recommendation to provide sufficient safety-related instrumentation (able to withstand design basis natural phenomena) to monitor from a control room the key parameters of the spent fuel pool (e.g., temperature, radiation level, etc.). Monitoring has been identified by DOE as a high priority crosscutting need. Monitoring is necessary to determine and predict the degradation state of the systems, structures, and components (SSCs) important to safety (ITS) and is required by regulation (10 CFR 72.122 and 10 CFR 72.128). Revisions to NUREG 1927 [2] suggest requirements for monitoring and inspection of dry storage systems as part of aging management plans. To ensure that nuclear power remains clean energy, safe, long-term management of used nuclear fuel and high level radioactive waste "remains a national priority".

Therefore, nondestructive structural material degradation and condition monitoring is in urgent need and must be integrated into the fuel cycle to quantify the "state of health", and more importantly, to guarantee the safe operation of existing nuclear power plants (NPP) and radioactive waste storage systems (RWSS) during their predicted life span. Innovative approaches are desired to evaluate degradation and aging phenomena of used fuel containers and storage facilities under extended storage. To meet the ever-growing awareness of nuclear safety, a state-of-the-art nuclear structural health monitoring (N-SHM) system is necessary that uses in-situ sensing technologies to monitor material degradation and aging for DCSS canister and similar structures (e.g., wet storage pools), as conceptually illustrated in Figure 1.

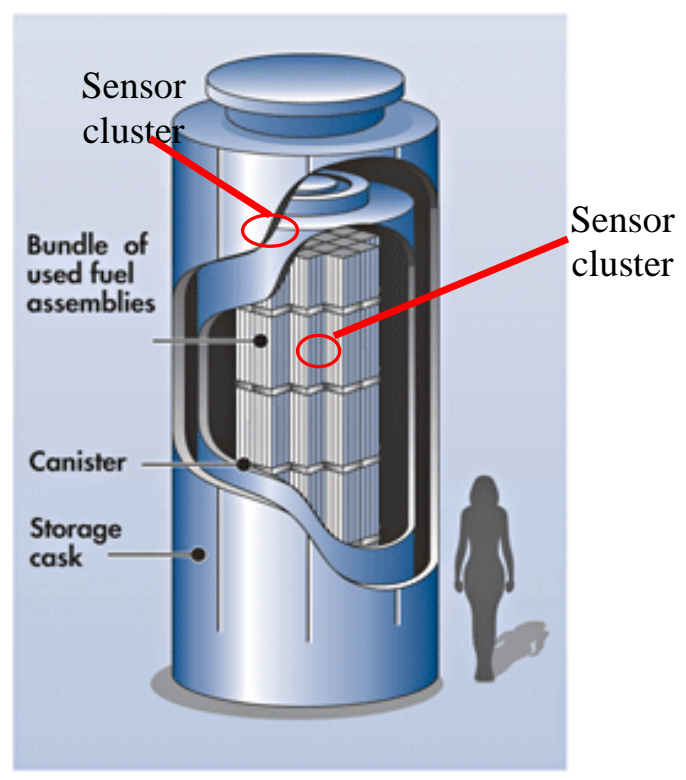

Fig 1: Sensor cluster on typical dry cask storage system (http://www.nrc.gov/waste/spent-fuel-storage/diagram-typicaldry-cask-system.html)

The key technology to an effective N-SHM system is the sensing element that can detect the degradation under the harsh nuclear DCSS environment. The past decades have witnessed an extensive development of SHM sensor technology [3]-[5]. A wide range of sensors have been developed particularly for generating and receiving acousto-ultrasonic waves. Common examples of such SHM sensors are the piezoelectric wafer active sensor (PWAS) transducers [3] and the fiber Bragg grating (FBG) optical sensors [6]. The study on PWAS that exposed to radiation was performed in our previous research. The PWAS irradiation study not only provides the fundamental understanding of the PWAS irradiation survivability but also tests the potential of PWAS as irradiation sensors for nuclear applications [7]. A combined modeling and experimental study on assessing nuclear effects on the PWAS acousto-ultrasonic system to identify and quantify the possible influences of the nuclear environment typical of DCSS (temperature and radiation) was used to develop adequate solutions and guidelines accordingly [8], [9]. However, the irradiation and temperature effect on FBG sensors for DCSS application have not been studied. In this paper, we will focus on the temperature effect on FBG spectrum, FBG ultrasonic guided waves sensing. An automatic FBG center wavelength tracking system was introduced to compensate the temperature effect on the guidedwaves. 


\section{FIBER BRAGG GRATING SENSORS}

Fiber optics sensors have known extensive development for SHM applications. Optical fibers consist of a very small inner core (which has a high reflection index caused by germanium doping) and an outer part of pure glass with a smaller reflection index. Total internal reflection takes place due to the large difference in the reflection indices. The FBG sensor (Fig 2a) is a permanent periodical perturbation (grating) in the index of refraction of the optical fiber core inscribed at selected locations using high-intensity UV light. This periodic perturbation with pitch $\Lambda$ acts as a wavelength filter with a narrowband reflection spectrum centered on the Bragg wavelength $\lambda_{B}=2 \eta_{\text {eff }} \Lambda$ (Fig 2b). When mechanical strain $\Delta \varepsilon$ and temperature change $\Delta T$ are present, the Bragg wavelength shifts is

$$
\Delta \lambda_{B}=\Delta \lambda_{B S}+\Delta \lambda_{B T}=\lambda_{B}\left(1-\rho_{\alpha}\right) \Delta \varepsilon+\lambda_{B}(\alpha+\xi) \Delta T
$$

where $\xi$ is the thermo-optic coefficient and $\rho_{\alpha}$ is the effective strain-optic coefficient. If the temperature is constant, only the effect of strain is present. Thus, an FBG sensor bonded to a structural surface would respond to the structural strain by shifting its spectrum.
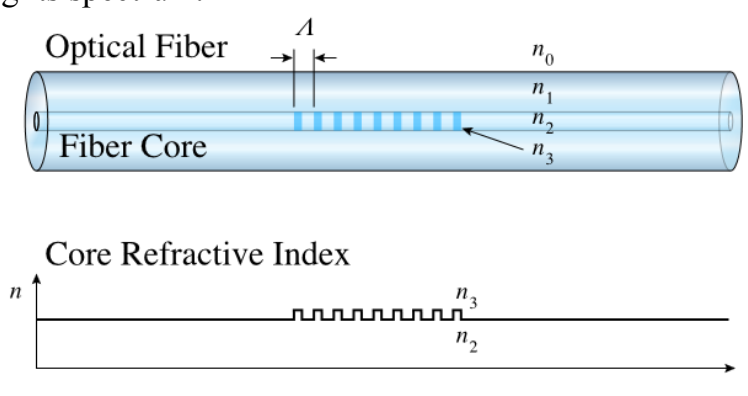

(a)

(b)

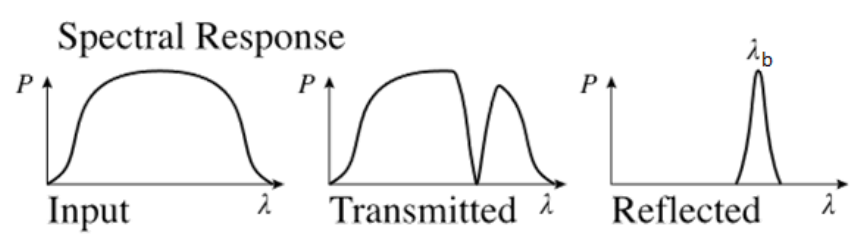

Fig 2: (a) Principles of fiber Bragg grating (FBG) optical sensors; (b) details showing the notch in the transmission spectrum and the peak in the reflection spectrum at the Bragg wavelength $\lambda_{B}$.

Fiber optics sensors offer several advantages over piezoelectric sensors for SHM applications: (a) immunity to electromagnetic interference (EMI); (b) corrosion resistance; (c) the promise of direct embedment into the composite material along with the reinforcing fibers; (d) capability of working in wet and/or underwater environments, etc. In addition, FBG sensors offer the possibility of multiplexing several sensors of slightly different wavelength on the same optical fiber and interrogating them individually. The methods used for the demodulation and interpretation of the optical signal are very diverse and still evolving.

\section{FBG STRAIN SENSING}

The methods for FBG interrogation include wavelength division multiplexing (WDM), time division multiplexing (TDM), and optical frequency domain reflectometry (OFDR) which rely on various types of wavelength dependent filtering. Based on the light source used in the system, FBG system can be classified as two types: (1) broadband light emitting diode (LED) source; (2) narrow linewidth laser source. In broadband LED source FBG systems, a wavelength detection subsystem is required to demodulate the FBG sensor signal. In recent years, many commercial off-the-shelf (COTS) FBG interrogators have come to market but typically, regardless of the demodulation approach, they tend to compromise between speed, multiplexing capability, and strain resolution. Most FBG interrogation system uses broadband laser sources because of the high cost of scanning laser source. A summary of the available commercial FBG interrogators for dynamic sensing applications is given in ref [14]. Lin, et al. [15] evaluated several new versions of FBG interrogation systems available on the market (Micron Optics SM690 and Redondo Optics M200) for SHM applications. These studies focused on the frequency ranges, strain resolutions, and noise. From previous comparative study, it was apparent that most commercial FBG systems were based on WDM and were not good for low-amplitude ultrasonic measurements. Wild et al. [16] reviewed acoustic and ultrasonic optical sensors including FBG.

In narrow-linewidth laser source FBG systems, the narrowlinewidth laser reflected intensity is modulated by the FBG spectrum. If a narrow-linewidth laser is tuned to a wavelength positioned within the FBG reflective spectrum, the reflected amplitude is modified based on the FBG spectrum. A tunable laser source provides more capacity for multiplexing and less system noise than a broadband source; however, it is more expensive. Work on FBG for ultrasonic measurements began in 1996 [17] for sensing ultrasonic fields for medical applications. Perez et al. [18] used FBGs to detect acoustic emissions. They used a tunable matching FBG for demodulation. They successfully detected acoustic emissions generated by a piezoceramic resonator, an ultrasonic transducer, and a pencil lead breaking. Fomitchov and Krishnaswamy [19] studied the use of a FBG for the detection of ultrasonic waves in liquids and solid structures. Their system uses a tunable laser tuned to the full width half maximum (FWHM) point of the FBG, and a photodetector to measure the reflected intensity. They report sensitivity over a broad frequency range from $10 \mathrm{kHz}$ to $5 \mathrm{MHz}$. Lin et al. [11]-[13] developed an automated intensity-based system using the same method. Their system was capable of scanning of FBG arrays with nano-strain sensitivity (1 nanostrain resolution) and frequency response ( $\mathrm{MHz}$ sampling). 


\section{TEMPERATURE EFFECT ON FBG SPECTRUM}

Environmental variability of a nuclear-SHM system includes changes in both the sensors and the sensing methodology (AE, GUW, and EMIS). In this section, the temperature effect is focused on FBG sensors bonded on the aluminum structure.

The FBG was $10-\mathrm{mm}$ long with more than $90 \%$ reflectivity supplied by from AtGrating Technologies (http://www.atgrating.com/en/productview.asp?id=63). It is made from acrylate fiber (SMF-28e) with acrylate recoating. FBG sensor is located at the customized position (1-meter from one FC/APC connector, another 1-meter as pigtail). The FBG is apodized with a center wavelength at $1550 \mathrm{~nm}$ at room temperature. The specification is shown in Table 1.

Table 1: FBG specifications (www.atgrating.com)

\begin{tabular}{|c|c|c|c|c|c|}
\hline Parameters & Unit & \multicolumn{4}{|c|}{ Values } \\
\hline $\begin{array}{l}\text { Center } \\
\text { Wavelength }\end{array}$ & $\mathrm{nm}$ & \multicolumn{4}{|c|}{$1510 \sim 1590$} \\
\hline FBG Profile & & \multicolumn{4}{|c|}{ Apodized } \\
\hline $\begin{array}{l}\text { Wavelength } \\
\text { Tolerance }\end{array}$ & $\mathrm{nm}$ & \multicolumn{4}{|c|}{$+/-0.5$} \\
\hline FBG Length & $\mathrm{mm}$ & 3 & 5 & 10 & 15 \\
\hline Reflectivity & & $\geq 70 \%$ & $\geq 75 \%$ & $\geq 90 \%$ & $\geq 90 \%$ \\
\hline $\begin{array}{l}\text { Bandwidth } \\
\text { at }-3 \mathrm{~dB}\end{array}$ & $\mathrm{~nm}$ & $\leq 0.7$ & $\leq 0.7$ & $\leq 0.3$ & $\leq 0.3$ \\
\hline SLSR & $\mathrm{dB}$ & \multicolumn{3}{|c|}{$\geq 10$} & $\geq 15$ \\
\hline $\begin{array}{l}\text { Operating } \\
\text { Temp. }\end{array}$ & ${ }^{\circ} \mathrm{C}$ & \multicolumn{4}{|c|}{ SMF-28 fiber: $-40 \sim 120$} \\
\hline
\end{tabular}

The FBG reflective spectrum measurement equipment consists of a LUNA Phoenix 1400 tunable laser source (TLS), and an optical circulator (AFW Technologies Pty Ltd, \#CIR-315-L-1-2). The Phoenix 1400 is a swept narrow linewidth laser source covering the full C-band tenability $(1515 \mathrm{~nm}-1565 \mathrm{~nm})$ with two built-in optical power meters. The laser output connects to the circulator port 1 to provide a narrow-linewidth constant power to the port 2 on the circulator. The FBG sensors connect to the circulator port 2. When the TLS is swept at different wavelength within FBG spectrum, the FBG reflects the narrowwidth laser with intensity corresponds to its reflected spectrum. The reflected light goes through the circulator port 3 to the power meter (Fig 3). The temperature was recorded through a NI USBTC01 device connected to a K-type thermocouple.

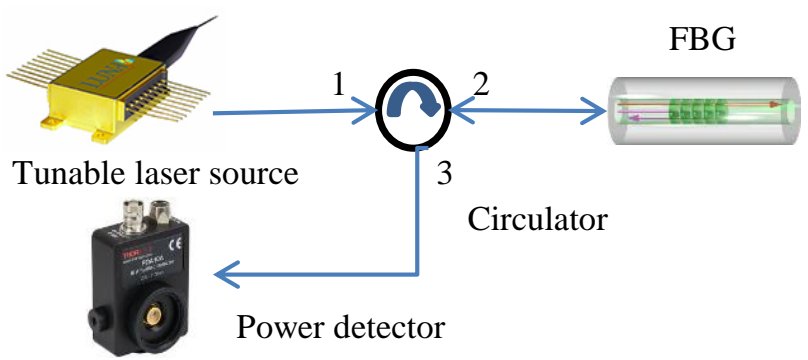

Fig 3: Basic setup of FBG spectrum measurement

One FBG was bonded on an aluminum panel using Vishay Measurement Group Mbond-200 adhesive (Fig 4). The FBG spectra at different temperature were recorded (Fig 5). We found that the thermal expansion of the aluminum substrate stretch the FBG grating and in turn to increase the center wavelength of the FBG. The reflected peak amplitude has not changed much. The center wavelength and reflective peak power of the FBG is listed in Table 2.

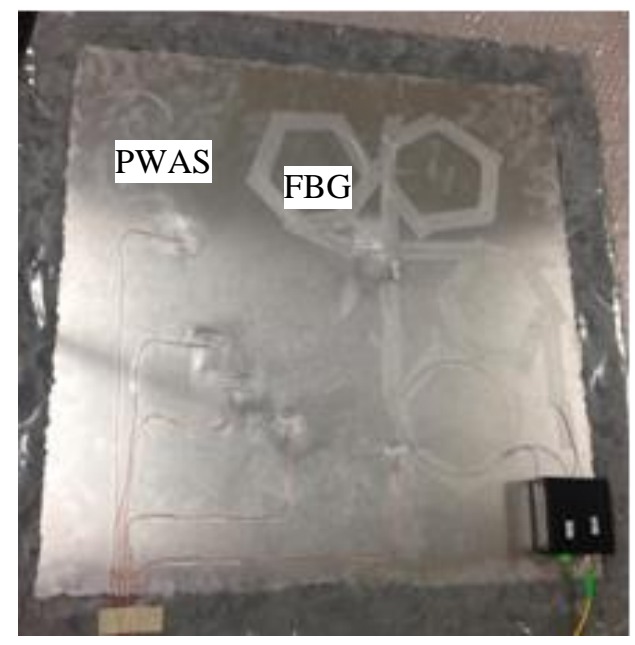

Fig 4: Basic setup of FBG spectrum measurement

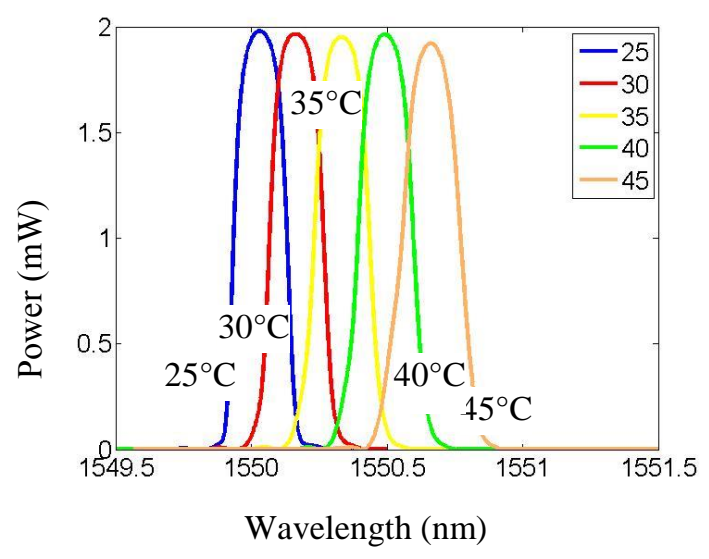

Fig 5: Spectra of a FBG bonded on an aluminum panel. 
Table 2: FBG center wavelength / power vs. temperature

\begin{tabular}{ccc}
$\mathrm{T}\left({ }^{\circ} \mathrm{C}\right)$ & Center wavelength $(\mathrm{nm})$ & Peak power $(\mathrm{mW})$ \\
\hline 25 & 1550.031 & 1.980 \\
30 & 1550.169 & 1.965 \\
35 & 1550.330 & 1.951 \\
40 & 1550.489 & 1.965 \\
45 & 1550.663 & 1.922 \\
\hline
\end{tabular}

From the date in Table 2, we have found that the center wavelength of FBG shift linearly with the temperature (Fig 6). The ratio is $31.7 \mathrm{pm} /{ }^{\circ} \mathrm{C}$. Consider the $\mathrm{FBG}$ is bonded to the substrate; we ignore the thermal effect on the FBG itself since it is mainly constrained by the substrate strain change. At the center wavelength at $1550 \mathrm{~nm}$, the strain to wavelength ratio for the FBG is $1.2 \mathrm{pm} / \mu \varepsilon$. So the measured thermal expansion coefficient for the aluminum substrate is $26.4 \mu \varepsilon /{ }^{\circ} \mathrm{C}$.

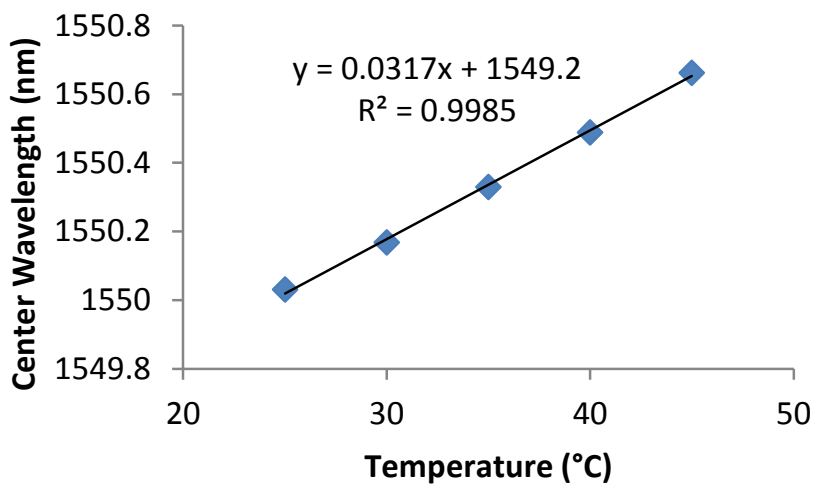

Fig 6: FBG center wavelength is linear with the temperature change.

\section{TEMPERATURE EFFECT ON PIEZO-OPTICAL SENSING}

The common way of using the FBG sensor for strain measurements is to track the spectral shift $\Delta \lambda_{B}$ of the reflected signal and convert it into strain change $\Delta \varepsilon$. However, this type of demodulation is only effective for sizable strain values (say, several $\mu \varepsilon$ ) and is not effective for the very small strains encountered in ultrasonics wave propagation which are several order of magnitude smaller $(0.010-0.100 \mu \varepsilon)$. The demodulation method used for the detection of such small strains is based on up and down excursions from the midpoint of spectral slope (Fig 7). This so-called FWHM (full-width halfmaximum) method uses a narrow-band tunable laser source precisely positioned on the FWHM point of the spectrum and several optical components to direct the reflected optical signal to a low-noise photo detector where is converted into an electrical signal that can be fed into an oscilloscope for display and digitization. To be able to track a small wavelength change due to a small strain, the FBG spectrum slope needs to be high. This limits the total sensing range of the FBG sensors. The typical linear region of the FBG slope is only $50 \mathrm{pm}$, which corresponds to the total $40 \mu \varepsilon$. As shown in the previous section, a $1{ }^{\circ} \mathrm{C}$ variation in temperature will change the $\mathrm{FBG}$ center wavelength of $32 \mathrm{pm}$.

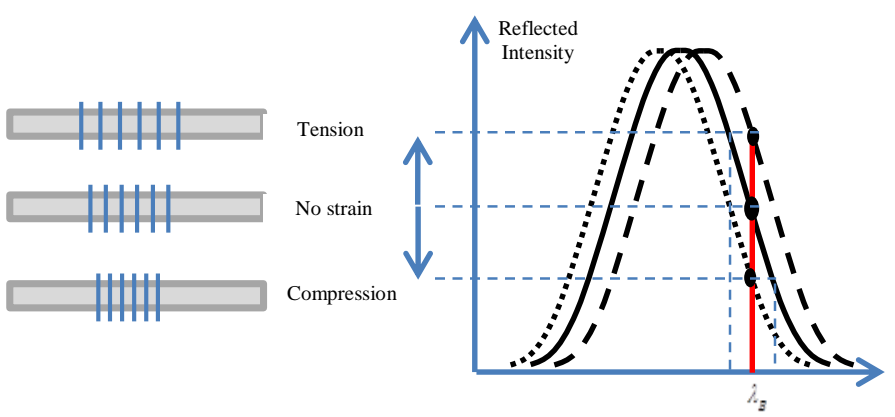

Fig 7: Principle of an operation of intensity-based full-width halfmaximum (FWHM) FBG interrogation system [11]

In the same experimental setup as shown in Fig 4, we bonded one PWAS actuator to excite the structure. The excitation PWAS was connected of an HP 33120A signal generator and was excited with $20 \mathrm{Vpp}, 100 \mathrm{kHz}$, three-count tone-burst signal. For the pitch-catch experiments, the output signals from the FBG receiver were sent to one channel of a Tektronix TDS5034B digital oscilloscope that also served as signal digitizer. At the room temperature $\left(24^{\circ} \mathrm{C}\right)$, the TLS was tuned to the falling slope of the FBG spectrum as shown in the Fig 7. FBG sensor was oriented along the wave propagation direction. The distance between the PWAS and FBG was $200 \mathrm{~mm}$. Both non-dispersive $\mathrm{S} 0$ and dispersive A0 Lamb wave modes were captured by the FBG sensor.

As shown in Fig 8, FBG collected both $\mathrm{S} 0$ and A0 signal at $24^{\circ} \mathrm{C}$ with maximum amplitude since the TLS is tuned at the maximum slope point (FWHM point). At $26^{\circ} \mathrm{C}$, the TLS source is still fixed at its original wavelength, the $\mathrm{FBG}$ center wavelength shifted toward right in $64 \mathrm{pm}$, this move the FBG slope is small than the FWHM point, both $\mathrm{S} 0$ and $\mathrm{A} 0$ signal is reduced. At $28^{\circ} \mathrm{C}$, the $\mathrm{FBG}$ spectrum continued moving up. The TLS fixed point now reached the top of flat spectrum. There is no S0 and A0 signals were recorded. At $30^{\circ} \mathrm{C}$, the $\mathrm{FBG}$ spectrum shifted around $200 \mathrm{pm}$ which is the bandwidth of the FBG. TLS is now at the middle point of the rising slope, we received the maximum amplitude of the $\mathrm{S} 0$ and $\mathrm{A} 0$ signal again. However, the signal is inverted with $180^{\circ}$ phase change. Continue to raise the temperature, the $\mathrm{S} 0$ and $\mathrm{A} 0$ signal decrease again at $32{ }^{\circ} \mathrm{C}$ and completely disappeared at $35^{\circ} \mathrm{C}$. From this simple experiment, we can conclude that the small environmental temperature change will change the performance of the FBG capability of tracking small ultrasonic guided waves. An appropriate temperature feedback is necessary to adjust the FBG ultrasonic sensing. 


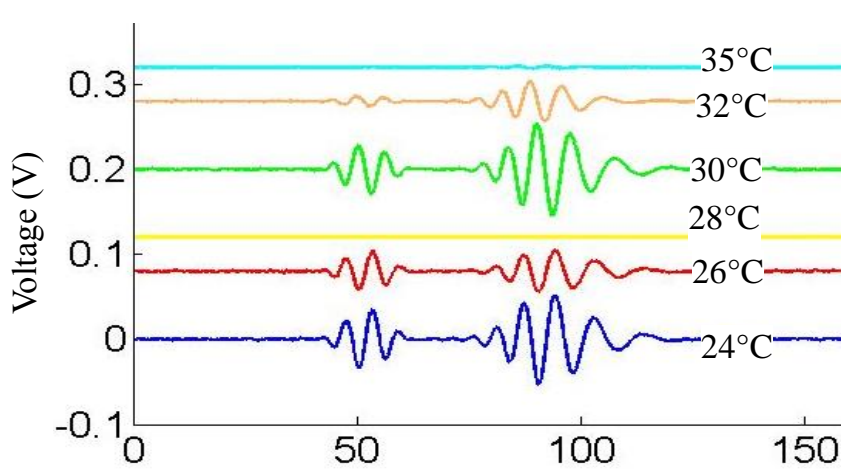

Time $(\mu \mathrm{s})$

Fig 8: The recorded pitch-catch waveform at different temperatures with the TLS was fixed at FBG spectrum falling slope at the room temperature.

As we noticed that the piezo-optical sensing is very sensitive to the small temperature changes. We used the temperature as the feedback to adjust the TLS source locking wavelength. The TLS source is not fixed to a certain wavelength. Instead, the TLS locking wavelength is tracking with the temperature and always following the falling slope of the FBG spectrum. In this way, the pitch-catch signal received from the $\mathrm{FBG}$ is similar. Fig 9 showed the recorded pitch-catch waveform from $25^{\circ} \mathrm{C}-45^{\circ} \mathrm{C}$. The peak to peak amplitude and waveform is almost identical at all measured temperature.

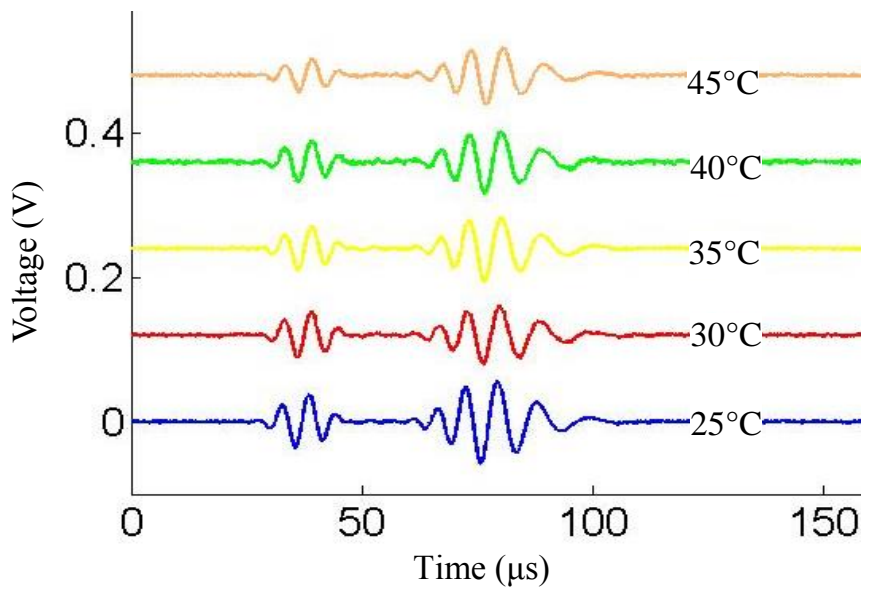

Fig 9: The recorded pitch-catch waveform at different temperatures with the TLS was automatically tuned at FBG spectrum falling slope at the corresponding temperature.

\section{SUMMARY AND CONCLUSIONS}

SHM is a multidisciplinary process that involves several disciplines that must be closely coordinated. Sensors are being used to measure parameters such as temperature, pressure, radiation levels, $\mathrm{pH}$, and wall thickness or to indicate that damage or failure in a system has already occurred (i.e. detection of a leak). But through the implementation of SHM approach the detection of materials degradation at relatively early stages, before the damage occurs, can be potentially achieved. Therefore, the development of active and passive nondestructive evaluation methods based on SHM provides an opportunity to progress the capability of monitoring DCSS and similar PVP systems. The integration of SHM with existent NDE tools in nuclear fields can increase the confidence of the safe operation and provide assurance of in-service reliability. Moreover, the development of SHM technologies can minimize human intervention, decrease the cost associated with NPP operation, and improve the reliability of essential systems by continuously assessing the structural integrity of nuclear related facilities. While SHM have been employed in different fields, its applicability for PVP systems will require further development and evaluation. This will required research to address some of the challenges that were discussed. SHM monitoring can provide decision makers, regulatory agencies, and PVP system operators with timely information on the health of the system. Ultimately, this information will result in the reduction of operation and maintenance cost, and the timely response can protect civilian population from catastrophic system failure.

We address the fundamental issue of optical ultrasonic sensing using FBG sensors. The piezo-optical sensing is very sensitive to the small temperature changes as we discovered in this paper. A feedback algorithm is also provided to compensate the temperature effect. We have proved that the compensating method is also critical to the accuracy of the experimental result for FBG sensing. This preliminary study paved the road to use the FBG sensors to use in the harsh environment. The potential of FBG sensors for DCSS applications was explored. The future work should also consider other nuclear effect in additional to the thermal effect to the FBG sensor and sensing methodology to provide robust monitoring of the health of DCSS and other PVP applications.

\section{ACKNOWLEDGEMENT}

This material is based on work supported by Office of Naval Research grant \# N00014-11-1-0271, Dr. Ignacio Perez technical representative; by Department of Energy award DE-NE0000726, program manager Mr. Kenny Osborne, and by project APSEM Skolkovo from Skoltech Institute of Science and Technology. Any opinions, findings, and conclusions or recommendations expressed in this material are those of the authors and do not necessarily reflect the views of the Office of Naval Research, the Department of Energy, and Skoltech Institute of Science and Technology . 


\section{REFERENCE}

[1] NRC 10 CFR: US Nuclear Regulatory Commission Regulations: Title 10, Code of Federal Regulations, Part 72: Licensing requirements for the independent storage of spent nuclear fuel and high-level radioactive waste, and reactorrelated greater than Class $\mathrm{C}$ waste, http://www.nrc.gov/reading-rm/doc-collections/cfr/ accessed Jan 2014

[2] Nureg-1927, Standard review plan for renewal of spent fuel dry cast storage system licenses and certificates of compliance final report, http://www.nrc.gov/reading-rm/doccollections/nuregs/staff/sr1927/ Mar, 2011

[3] Giurgiutiu, V. (2008) Structural Health Monitoring with Piezoelectric Wafer Active Sensors, Elsevier Academic Press, 760 pages, ISBN 978-0120887606, 2008

[4] Balageas, D. et al., (eds.), Structural Health Monitoring, ISTE (2006).

[5] Alleyne, D.N. "The Nondestructive Testing of Plates Using Lamb Waves", Mechanical Engineering Department, Imperial College of London, London, (1991).

[6] Peters, K. (2008) "Fiber Bragg Grating Sensors" in Encyclopedia of Structural Health Monitoring, Boller, C;Chang, F-K;Fujino, Y, Eds., ed: Wiley, 2008

[7] Lin, B.; Mendez-Torres, A. E.; Gresil, M.; Giurgiutiu, V. (2012) "Structural Health Monitoring with Piezoelectric Wafer Active Sensors Exposed to Irradiation Effects", ASME 2012 Pressure Vessels and Piping Division Conference, 15-19 July 2012, Toronto, Ontario, Canada, paper PVP2012-78848

[8] Lin, B.; Gresil, M.; Giurgiutiu, V.; Knight, T.; Mendez-Torres, A.; Yu, L.; (2014) "Nuclear environmental effects on piezoelectric wafer active sensors based acousto-ultrasonic sensing system" 2014 International Congress on Advances in Nuclear Power Plants, ICAPP 2014, April 6-9, 2014 Charlotte, NC, paper No.14304

[9] Lin, B.; Yu, L.; Giurgiutiu, V.; (2014) "Analytical model of nuclear environmental effects with piezoelectric wafer active sensors", Proceedings of the ASME 2014 Pressure Vessels \& Piping Division Conference, PVP 2014-28887, July 20-24,
2014, Anaheim, CA

[10] Doctor, S.R. (2007), "Nuclear Power Plants NDE Challenges: Past, Present and Future", Review of Quantitative Nondestructive Evaluation, Vol. 26, 2007

[11] Lin, B.; Giurgiutiu, V. (2014) "Development of optical equipment for ultrasonic guided wave structural health monitoring", SPIE 2014 Smart Structures and NDE, 9-13 March 2014, San Diego, CA, SPIE Vol. 9062, paper \# 906227

[12] Giurgiutiu, V.; Roman, C.; Lin, B.; Frankforter, E.; (2015) "Omnidirectional FBG Ring Sensor for Enhanced Guided Wave Structural Health Monitoring", Smart Materials and Structures, 24, 015008, doi:10.1088/0964-1726/24/1/015008

[13] Lin, B.; Giurgiutiu, V. (2014) "Piezo-Optical Active Sensing With PWAS And FBG Sensors For Structural Health Monitoring" ASME 2014 Conference on Smart Materials, Adaptive Structures and Intelligent Systems, Newport, Rhode Island, Sep 8-10, 2014 SMASIS2014-7581

[14] Norman, P.E.; Davis, C.E. (2010) "An Intensity-based Demodulation Approach for the Measurement of Strains Induced by Structural Vibrations Using Bragg Gratings", DSTO Defense Science and Technology Organization, Australia, DSTO TR-2370

[15] Lin, B.; Giurgiutiu, V. (2013) "Exploration of Ultrasonic Guided Wave Detection with Optical Fiber Sensors and Piezoelectric Transducers", 9th International Workshop on Structural Health Monitoring, IWSHM, Sept. 10-12 2013, Stanford University, CA, pp. 1559-1566

[16] Wild, G. (2008) "Acousto-Ultrasounic Optical Fiber Sensors: Overview and State-of-the-Art", IEEE Sensors Journal, vol. 8, no.7, July 2008

[17] Webb, D. J., et al., (1996) "Miniature fiber optic ultrasonic probe," Proc. SPIE, vol. 2839, pp. 76-80, 1996.

[18] Perez, I.; Cui, H. L; Udd, E. (2001) "Acoustic emission detection using fiber Bragg gratings," Proc. SPIE, vol. 4328, pp. 209-215, 2001

[19] Fomitchov, P. A.; Krishnaswamy, S. (2003) "Response of a fiber Bragg grating ultrasonic sensor," Opt. Eng., vol. 42, no. 4, pp. 956-963, 2003 